\title{
Leere Versprechungen oder die Lösung globaler Herausforderungen?
}

\author{
Mit der zunehmenden Digitalisierung der \\ Wirtschaft wird der Verbrauch von speziellen \\ Metallen und Seltenen Erden steigen. \\ Welche sozialen und ökologischen Folgekosten \\ sind damit in globaler Perspektive verbunden? \\ Von Michael Reckordt
}

D ie deutsche Wirtschaft ist zu nahezu 100 Prozent von Primärmetallimporten abhängig. Für Produkte „Made in Germany“ werden Niob aus Brasilien, Bauxit aus Guinea, Seltene Erden aus China, Nickel aus den Philippinen, Kupfer aus Peru oder Kobalt aus der Demokratischen Republik Kongo benötigt. Bei vielen metallischen Rohstoffen gehört Deutschland zu den größten globalen Verbrauchern, sowohl für die Produktion von Gütern als auch bei deren Konsum. Laut UN-Comtrade-Statistiken wurden im Jahr 2016 mehr als 66 Millionen Mobiltelefone und knapp 25 Millionen Laptops importiert, in denen schon viele der genannten Rohstoffe verarbeitet sind. Zukünftig wird mit einem weiter ansteigenden Verbrauch von metallischen Rohstoffen gerechnet. So erwartet das Umweltbundesamt (UBA), dass die deutsche Wirtschaft 2030 das Dreifache an Aluminium und Zink sowie das Doppelte an Kupfer, Chrom, Gold und Platin im Vergleich zum Jahr 2010 verbrauchen wird (UBA 2015). Durch die Digitalisierung der Wirtschaft im Zuge von Industrie 4.0 kann der Verbrauch von $\mathrm{Zu}$ kunftsmetallen wie Seltenen Erden oder Lithium noch dramatischer steigen. Die sozialen und ökologischen Folgekosten treten nur zweitrangig in Deutschland auf. Sie werden vor allem externalisiert; die Bewältigung bleibt den Gemeinschaften in den Abbauregionen überlassen.

\section{Diskurs ist von Industrie dominiert}

Seit einigen Jahren versprechen Wirtschaft, Politik und Forschungsinstitute die vierte Welle der industriellen Revolution. Im angelsächsischen Raum wird vom „Internet of Things“ gesprochen. In Deutschland firmiert die Digitalisierung der Fertigung und des Vertriebs unter dem Begriff „Industrie 4.0“. Für den Bundesverband der Deutschen Industrie (BDI) ist Industrie 4.0 ein radikaler Strukturwandel: „Neue Daten, Vernetzung, Automatisierung und die digitale Kundenschnittstelle sprengen bestehende Wertschöpfungsketten“. Industrie 4.0 sei die Neukonfigurierung des globalen Produktionssystems oder gar eine „Reindustrialisierung“. Der BDI rechnet, dass ein Zuwachs von 1,25 Billionen Euro an industrieller Bruttowertschöpfung bis 2025 möglich ist, warnt aber zugleich vor möglichen Verlusten in Höhe von 605 Milliarden Euro in der Wertschöpfung, wenn nicht die passenden Weichen gestellt werden. Industrie 4.0 ist also das Versprechen, Exportweltmeister bleiben zu können, wenn nur die Chancen genutzt werden.

Es sind vor allem große Wirtschaftsverbände aus dem Bereich Elektronikindustrie (ZVEI), Maschinen- und Anlagenbau (VDMA) sowie der Verband der digitalen Industrie (BITKOM), die, unterstützt von der Bundespolitik, den Diskurs der Industrie 4.0 und entsprechende Umsetzungsprozesse vorantreiben. Alle drei Verbände sind wichtige Mitglieder im BDI und haben die Plattform Industrie 4.0 initiiert. „Bundeswirtschaftsministerin Brigitte Zypries und Bundesforschungsministerin Prof. Dr. Johanna Wanka steuern und leiten die Plattform gemeinsam mit hochrangigen Vertretern aus Wirtschaft, Wissenschaft und Gewerkschaften", steht auf der Homepage der Plattform. Zwar arbeitet das von der Industrie dominierte Bündnis in der Plattform mit einigen Forschungsinstituten und der Gewerkschaft IG Metall zusammen, eine Beteiligung von Umweltoder Entwicklungsverbänden findet aber nicht statt. Dadurch fehlen ökologische, menschenrechtliche und entwicklungspolitische Ansätze nahezu vollständig im bisherigen Diskurs.

\section{Die industrielle Veränderung wirkt gewaltig}

Eine vom Bundeswirtschaftsministerium (BMWi) im Jahr 2015 erarbeitete Zukunftsvision geht von Veränderungen auf unterschiedlichen Ebenen aus: Dazu gehören neue Marktchancen und Exportmöglichkeiten, eine nachhaltigere Wirtschaft inklusive Ressourcenschonung und Energieeffizienz, Entstehung von qualitativ hochwertigen Arbeitsplätzen, neue Freiräume und soziale Teilhabe sowie eine steigende Lebensqualität, „weil die Digitalisierung zum Nutzen der Menschen eingesetzt wird“ (BMWi 2015). Das klingt so, als würden einige der drängenden Probleme der Zukunft bald gelöst werden.

Industrie 4.0 verspricht die Lösung bestimmter Krisen durch die Entwicklung neuer Technologien. Sie macht im Kern ein ähnliches Versprechen wie die Grüne Ökonomie. „Mit mehr technologischer Innovation schaffen wir die Effizienzrevolution und die Entkopplung der Wirtschaftsleistung vom Energieund Materialverbrauch“, fassen die Kolleg/innen der HeinrichBöll-Stiftung in ihrer „Kritik der Grünen Ökonomie“ die Ziele 
derselbigen zusammen (Fatheuer et al. 2015). Beiden Narrativen kann eine Technologiegläubigkeit unterstellt werden, die blind ist „für Fragen der Macht und Politik und für Fragen von Gerechtigkeit und Demokratie“ (Fatheuer et al. 2015).

Die Deutsche Rohstoffagentur (DERA) betont derweil, dass die für die Digitalisierung der Industrie benötigten Technologien wie Sensoren, Radio-Frequency-Identification(RFID)Tags, Industrie-Roboter etc. in hohem Maße metallische Rohstoffe verbrauchen. Allein für $42 \mathrm{Zukunftstechnologien,} \mathrm{so}$ schätzt die DERA, wird bis 2035 das Vierfache der heutigen Produktion an Lithium, das Dreifache an Schweren Seltenen Erden sowie das Anderthalbfache an Leichten Seltenen Erden und Tantal benötigt (DERA 2016). Da ist der steigende Verbrauch für heutige Technologien wie Kommunikationstechnologien, Transportmittel oder Häuserbau nicht mit einberechnet. Expert/innen gehen davon aus, dass die weltweite KupferNachfrage in den nächsten Jahren bis 2050 zwischen 213 und 341 Prozent zunehmen wird (Müller 2017).

\section{Keine Atempause, Geschichte wird gemacht: Es geht voran}

Diesem vollkommen verantwortungslosen Mehrverbrauch an metallischen Rohstoffen, der schon heute zu gewaltsamer Vertreibung, politischen Morden, Kriegsfinanzierung, gewalttätiger Niederschlagung von Protesten und ökologischen Katastrophen in den Abbauregionen führt, wird mit einem starken Glauben an den Fortschritt begegnet. So sprechen einige Vertreter/innen der Wirtschaft von einer Dematerialisierung durch die Industrie 4.0. In einer von der Deutschen Telekom finanzierten Publikation wird vorgerechnet, dass im Bereich der E-Paper - durch elektronische Zeitungen und Bücher - ein Einsparpotenzial von 9,5 Millionen Tonnen Papier bestünde, was einem theoretischen Reduktionspotenzial der Treibhausgase um 4,5 Megatonnen $\mathrm{CO}_{2}$-Emissionen entspräche (GeSi o. J.). Inwieweit die Produktion, der Strom und die End-of-Life-Phase der E-Reader und Computer, die man zum Lesen benötigt, einberechnet sind, ist jedoch offen. Und selbst wenn diese mitberechnet wären, sind metallische Rohstoffe im Gegensatz zu Holz nicht nachwachsend. Vielmehr werden die Metalle häufig in so kleinen Mengen als Legierungen, Lötpunkte etc. in den Elektronikprodukten aufgetragen, dass sie technisch nicht zurückgewonnen werden können.

Die Potenziale einer Dematerialisierung sind bisher nur an Einzelbeispielen analysiert und noch nicht systematisch zusammengetragen worden. Doch schon jetzt scheint es auch in der Industrie eine Skepsis gegenüber der Dematerialisierung zu geben. „Für die Technologien von morgen brauchen wir mehr Rohstoffe - für ein Elektroauto zum Beispiel $60 \mathrm{~kg}$ mehr Kupfer, $50 \mathrm{~kg}$ mehr Aluminium, $20 \mathrm{~kg}$ mehr Stahl und $10 \mathrm{~kg}$ mehr Nickel als für einen herkömmlichen Verbrenner. Bei Erneuerbaren Energien und der Telekommunikation spielen Aluminium, Kupfer, Magnesium, Nickel, Zink, Blei und andere Metalle ebenfalls eine wichtige Rolle“, schreibt z. B. die Wirt- schaftsvereinigung Metalle auf ihrer Homepage (WVM 2016). Der BDI wird noch deutlicher: „Ohne Rohstoffe keine Energiewende, keine Elektromobilität, keine schnellen Breitbandnetze, und keine Industrie 4.0“, schreibt er im Rahmen seines fünften Rohstoffkongresses mit dem Titel „Rohstoffsicherung 4.0“ im Jahr 2016 (BDI 2016).

Auch die von Politik und Industrie beschworene Ressourceneffizienz führt nicht zwangsläufig zu einer Verringerung des Verbrauchs. Wie uns die Forschung zu Rebound-Effekten zeigt, werden knapp 50 Prozent der Effizienzeffekte durch höheren Verbrauch der Mehrproduktion und stärkeren Konsum aufgefressen (Santarius 2013). Viele technologische Entwicklungen werden daher ohne politische Regulierung keine Dematerialisierung auslösen, sondern vor allem einen Wechsel von Rohstoffströmen: Fossile oder nachwachsende Rohstoffe werden vermehrt durch metallische Rohstoffe ersetzt. Der Ausstieg aus den Fossilen ist sicherlich zu begrüßen. Im Verkehrsbereich wird aber häufig eine Eins-zu-Eins-Ersetzung von Kraftfahrzeugen mit Benzin- oder Dieselantrieb durch elektrifizierte Kraftfahrzeuge diskutiert, was den Verbrauch von metallischen Rohstoffen massiv antreiben würde. Stattdessen sollten zukunftsfähige Verkehrskonzepte jenseits des Automobilismus eine größere Rolle in der Diskussion spielen, in denen Elektromobilität ein kleinerer Baustein sein könnte.

Ebenfalls ungeklärt ist bisher der Einfluss von Industrie 4.0 auf zunehmende Gefahren für Mensch und Umwelt durch Hackerangriffe auf Großindustrieanlagen, den Verlust von Arbeitsplätzen und Wertschöpfung in den Ländern des Globalen Südens sowie den Energieverbrauch der Digitalisierung von Billionen von Gegenständen. „Es gibt Schätzungen, dass von weltweit 200 Milliarden Objekten bis zum Jahr 2020 rund 32 Milliarden über das Internet vernetzt werden können“, schreibt das Wirtschaftsministerium (BMWi 2015). Bereits heute sind die Energieverbräuche von Servern und anderen Hardwaretechnologien gigantisch. Laut Greenpeace gehen sieben Prozent des weltweiten Energieverbrauchs auf den IT-Sektor zurück (Greenpeace 2016). Ein Trend, der durch die weitere Digitalisierung massiv zunehmen könnte. Industrie 4.0 birgt das Risiko, die planetaren Grenzen endgültig überzustrapazieren.

\section{Für eine global gerechte Rohstoffpolitik}

Umwelt-, Menschenrechts- und Entwicklungsorganisationen begleiten die Umsetzung von Industrie 4.0 und die flankierenden Maßnahmen bisher zu wenig. Es gibt allerdings erste Ansätze. Organisationen, die sich im AK Rohstoffe zusammengeschlossen haben, setzen sich für eine andere, global gerechtere Rohstoffpolitik ein. Sie fordern politische Maßnahmen, um den absoluten Rohstoffverbrauch zu reduzieren. Dazu gehören der Ausstieg aus den fossilen Energien, aber auch eine bessere Wiederverwendung, Reparierbarkeit und Recyclingfähigkeit. Im Zuge von einer auf Suffizienz ausgerichteten Politik sollte auf eine Beteiligung am Tiefseebergbau verzichtet werden. 
Der AK Rohstoffe fordert, dass die in Zukunft nach Deutschland kommenden Rohstoffe unter sozial und ökologisch bestmöglichen Bedingungen vor Ort gewonnen werden. Die Bundesregierung solle daher ein Sorgfaltspflichtengesetz verabschieden, das Unternehmen dazu verpflichtet, ihre Lieferketten auf menschenrechtliche Risiken zu überprüfen, diese zu minimieren und transparent über die Aktivitäten zu berichten. Bei einem Fehlverhalten solle dieses sanktionierbar sein. Zudem solle die Vergabe von Außenwirtschaftsförderung an menschenrechtliche Standards geknüpft sein. Auch in der Handelspolitik solle sich die Bundesregierung für Veränderungen einsetzen. Anstatt einseitige Klagerechte für Konzerne zu verankern, die in den letzten Jahren verstärkt von Bergbaukonzernen genutzt werden, um Umwelt- und Sozialauflagen zu unterlaufen, zu verwässern oder gar zu verhindern (Reckordt 2017), sollten stattdessen Menschenrechte, ILO-Kernarbeitsnormen und ökologische Standards in den Abkommen einklagbar verankert sein.

Industrie 4.0 bietet technische Möglichkeiten, die den Unternehmen helfen, den genannten Erwartungen zu begegnen. Schon heute können dank softwarebasierter Instrumente selbst komplexe Lieferketten nachverfolgt werden. Auch Informationen, die das Recycling und die Wiederverwendung von einzelnen Komponenten vereinfachen, könnten technologisch bereits sehr einfach umgesetzt werden. Doch die Erfahrungen der letzten Jahre mit freiwilligen Initiativen im Rohstoffsektor sind ambivalent. Daher muss die Politik anfangen, ihren Gestaltungsspielraum wieder zurückzugewinnen.

\section{Literatur}

BDI (2016): Ohne Rohstoffe keine Industrie 4.0; online unter: http://bdi.eu/ artikel/news/ohne-rohstof fe-keine-industrie-40

BMWi (2015): Industrie 4.0 und Digitale Wirtschaft - Impulse für Wachstum, Beschäftigung und Innovation.
DERA (2016): Rohstoffe für Zukunftstechnologien 2016.

Fatheuer, T./Fuhr, L./Unmüßig, B. (2015): Kritik der Grünen Ökonomie, oekom Verlag, München.

GeSI (o. J.): SMART 2020 Addendum Deutschland: Die IKT-Industrie als treibende Kraft auf dem Weg zu nachhaltigem Klimaschutz.

Greenpeace (2016): Clicking Clean: Wo is winning the race to build a green internet?; online unter: http://www.clickclean.org/germany/de/

Müller, M. (2017): Deutsche Kupferimporte: Menschenrechtsverletzungen, Unternehmensverantwortung und Transparenz entlang der Lieferkette. GLOCON Policy Paper, Nr. 1, Berlin.

Reckordt, M. (2017): Wenn Konzerne klagen können - Internationale Wirtschaftsabkommen schränken demokratische Rechte ein; in IZ3W \#358 Januar/Februar.

Santarius, T. (2013): Rebound Effekte vereiteln eine hinreichende Entkoppelung; online unter: http://www.postwachstum.de/rebound-effektevereiteln-eine-hinreichende-entkoppelung-20131021

UBA (2015): Global nachhaltige materielle Wohlstandsniveaus - Analyse und Veranschaulichung global nachhaltiger materieller Versorgungspfade auf der Ebene von Haushalten.

WVM (2016): Zugang zu internationalen Rohstoffmärkten gewährleisten und Handelsprotektionismus vermeiden. Online: http://www.wvmetalle.de/ geschaeftsfelder/handels-und-rohstoffpolitik/?L=\%25252525252525252 525252 Fproc\%25252525252525252525252Fself\%2525252525252525252 5252Fenviron

\section{AUTOR + KONTAKT}

Michael Reckordt arbeitet bei PowerShift als Koordinator des AK Rohstoffe, einem bundesweiten Netzwerk von Umwelt-, Menschenrechtsund Entwicklungsorganisationen, zur deutschen Rohstoffpolitik. Er hat zusammen mit Merle Groneweg und Hannah Pilgrim die Studie „Ressourcenfluch 4.0“ im Frühjahr 2017 veröffentlicht.

PowerShift e. V., Greifswalder Straße 4, 10405 Berlin. Tel.: +4930 42805479,

E-Mail: Michael.reckordt@power-shift.de

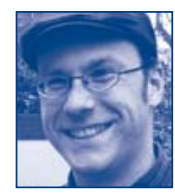

\section{Wo steht die Wirtschaft?}

Die Energiewende macht es vor, doch wie steht es um die Zukunftsfähigkeit in anderen Branchen? Expertinnen und Experten unterziehen Mobilität, Wohnen, Energie- und Agrarwirtschaft, Stahl- und Finanzbranche, Chemieindustrie und Maschinenbau einem Zukunfts-Check.
Heinrich-Böll-Stiftung

\section{Wirtschaft im Zukunfts-Check So gelingt die grüne Transformation}

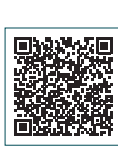

oekom verlag, München 240 Seiten, Broschur 19,95 Euro

ISBN: 978-3-96006-008-6

Im Handel ab dem: 04.05.2017

Auch als E-Book erhältlich

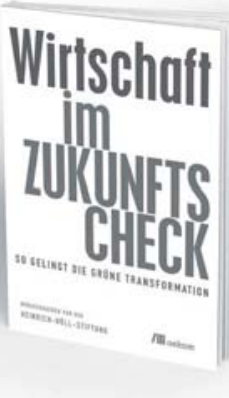

